\title{
Immunocytochemical and Pharmacological Evidence for GABAergic Spiking Local Interneurons in the Locust
}

\author{
A. H. D. Watson and M. Burrows \\ Department of Zoology, University of Cambridge, Cambridge CB2 3EJ, England
}

In the locust thoracic nervous system, spiking local interneurons within a ventral midline population are stained by a polyclonal antibody raised against GABA. Their cell bodies, their primary neurites in ventral commissure II, and their prominent neurites in the perpendicular tract linking ventral and dorsal fields of fine branches are all stained. Individual interneurons in this population were labeled with Lucifer yellow after their receptive fields had been determined physiologically. Alternate sections were stained with the antibody. Some, but not all, of the spiking local interneurons labeled with Lucifer yellow are also stained with the antibody. Apart from having somata that lie more posteriorly within the midline population, the antibody-stained interneurons cannot be distinguished on morphological or physiological grounds from those that are unstained. The way in which this cytochemical heterogeneity within an otherwise homogenous population might arise during development is discussed.

A second group of spiking local interneurons with similar sensory input to that of the ventral midline population, but with cell bodies in the anterior lateral region of the ganglion and primary neurites in ventral commissure $I$, are not stained.

Interneurons in the midline group receive direct inputs from sensory neurons and some directly inhibit particular leg motor neurons that mediate interjoint and tactile reflexes. Picrotoxin reversibly blocks the inhibition of the motor neurons and therefore abolishes these reflexes. Immunocytochemical and pharmacological experiments thus suggest that inhibition by some spiking local interneurons may be mediated by GABA.

The thoracic ganglia of arthropods have a considerable degree of autonomy in controlling the limbs of their segments, so that when an individual ganglion is isolated from the rest of the nervous system it can still mediate local reflexes evoked by touching hairs or moving joints (Burrows and Horridge, 1974; Sicgler and Burrows, 1986). A ganglion must therefore contain the necessary neurons to integrate sensory input and produce a coordinated motor output. In some reflexes, afferent neurons synapse directly with motor neurons-for example, those from hairplates (Pearson et al., 1976), a wing stretch receptor (Bur-

Received June 24, 1986; revised Sept. 24, 1986; accepted Oct. 2, 1986.

This work was supported by NIH Grant NS16058 to M.B. We thank our Cambridge colleagues for their constructive comments during the course of these experiments, K. J. Seymour for help with the pharmacological preparation, and N. J. Tublitz for assistance in preparing GABA-BSA conjugate. A.H.D.W. is a Beit Memorial Fellow.

Correspondence should be addressed to A. H. D. Watson, Department of Zoology, University of Cambridge, Downing Street, Cambridge CB2 3EJ, England. Copyright (C) 1987 Society for Neuroscience $0270-6474 / 87 / 061741-11 \$ 02.00 / 0$ rows, 1975), or a chordotonal organ (Burrows, 1987). Most reflexes, however, require the participation of local interneurons. In locust thoracic ganglia, spiking local interneurons are the primary integrators of mechanosensory information from the legs (Siegler and Burrows, 1983). Individual interneurons receive direct inputs from extero- and proprioceptors on particular parts of the leg (Burrows and Siegler, 1984; Burrows, $1985,1987)$. The organization of these interneurons is such that stimulation of a particular array of receptors leads to a specific local reflex movement (Siegler and Burrows, 1986). These interneurons have 2 distinct fields of branches within a ganglion, one ventral, which overlaps with the afferent terminals (Siegler and Burrows, 1983), the other dorsal and in a region of neuropile containing the neurites of leg motor neurons and nonspiking local interneurons (Siegler and Burrows, 1983, 1984). Some the the spiking local interneurons make direct inhibitory connections with motor neurons (Burrows and Siegler, 1982) and therefore form the central elements of 3-neuron reflex pathways. None have been found to make any excitatory connections.

Somata of one population of these spiking local interneurons lie in a position similar to those stained by antibodies raised against GABA (Watson, 1986), a known inhibitory transmitter in insects (Pitman, 1971). In this paper, we use intracellular injection of Lucifer yellow into physiologically characterized spiking local interneurons to demonstrate that some of them are stained by an anti-GABA antibody. Other spiking local interneurons that are similar on morphological physiological criteria are not stained by the antibody. The inhibition of certain motor neurons, mediated by spiking local interneurons during reflexes, is blocked by picrotoxin.

\section{Materials and Methods}

Immunocytochemistry. Adult Schistocerca gregaria (Forskal) were taken from our own crowded culture. The thoracic nervous system was fixed in situ for $5 \mathrm{~min}$ with $2.5 \%$ glutaraldehyde in $0.05 \mathrm{~m}$ phosphate buffer, $\mathrm{pH}$ 7.4. The thoracic ganglia were removed and fixed for a further $1-2$ $\mathrm{hr}$ in the same solution, then washed in phosphate buffer, dehydrated, and embedded in paraffin wax. Ten-micron serial sections were cut and stained using the procedure of Bishop and O'Shea (1982), which is a modification of the peroxidase-antiperoxidase (PAP) method of Sternberger (1974). The GABA antiserum, supplied by Dr. M. Geffard (Institut de Biochimie Cellulaire et Neurochimie, Bordeaux, France), was used at a dilution of 1:1600. It was raised in rabbit in response to scquential injections of GABA conjugated with glutaraldehyde to BSA, bovine hemoglobin, and poly(L-lysine). Details of the specificity of the antibody are described by Seguela et al. (1984). Goat anti-rabbit serum (IgG fraction) and rabbit PAP were obtained from Miles Scientific. 3,3diamino benzidine (Sigma) was used as substrate for the peroxidase reaction. The specificity of the antiserum was also tested by preabsorbing it with GABA conjugated to BSA, which was made using the method described by Seguela et al. (1984). A concentration of $500 \mu \mathrm{g} / \mathrm{ml}$ was 

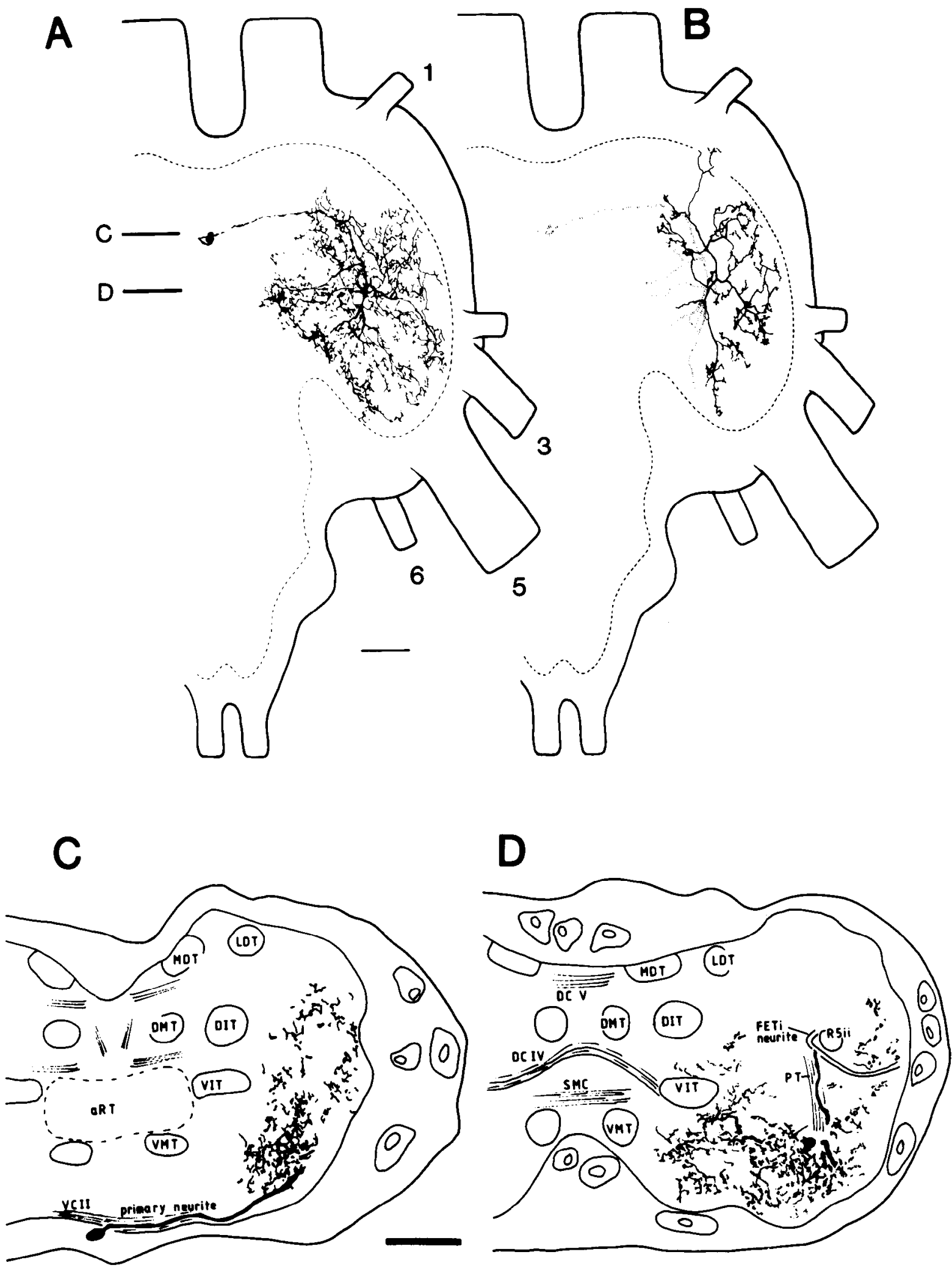

Figure 1. A spiking local interneuron in the midline group inhibiting the levator tarsi motor neuron. $A, B$, Whole-mount of a metathoracic ganglion, viewed dorsally, in which an interneuron was injected intracellularly with cobalt. The ccll body, primary ncurite, and ventral branches are shown in $A$, the more dorsal branches linked by a single process to the ventral ones in $B$. Lateral nerves $1,3,5$, and 6 are numbered; other nerves are omitted. The dashed line indicates the approximate extent of the neuropile. Anterior is at the top. The horizontal lines indicate the levels from which sections $C$ and $D$ are taken. $C$, Transverse section through a similar interneuron to showing the cell body and primary neurite in ventral commissure II. $D$, More posterior section showing the prominent process in the perpendicular tract. Scale bars, $100 \mu \mathrm{m}$. $a R T$, anterior ring tract; $D C I V, V$, dorsal commissures IV and V; $D I T$, dorsal intermediate tract; $D M T$, dorsal median tract; $F E T i$, fast extensor tibiae motor neuron; $L D T$, lateral dorsal tract; $M D T$, median dorsal tract; $P T$, perpendicular tract; $S M C$, superior median commissure; R5ii, root of nerve 5; VIT, ventral intermediate tract; $V M T$, ventral median tract. 


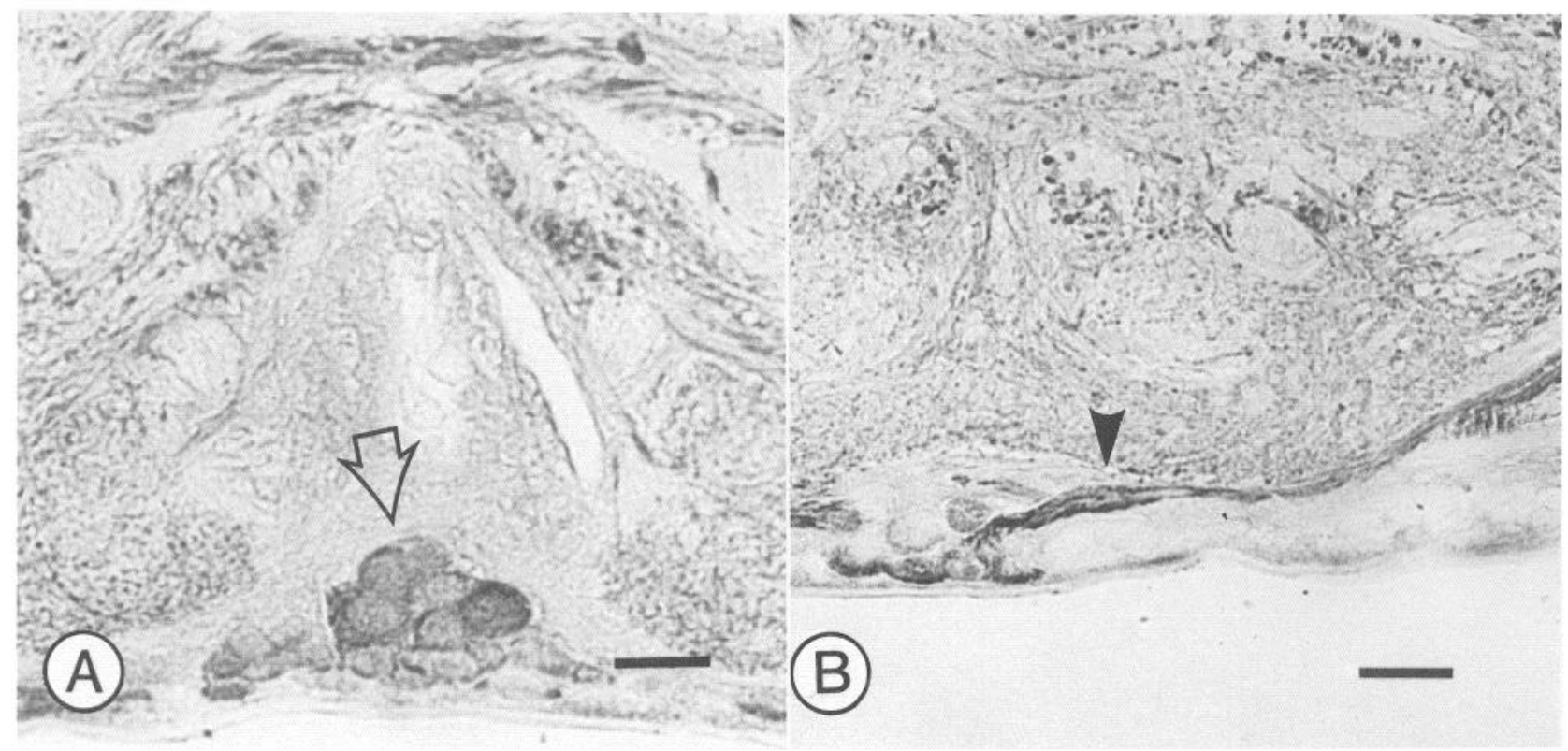

Figure 2. A, Cell bodies (arrow) at the ventral midline posterior to VCII in the mesothoracic ganglion exhibiting GABA-like immunoreactivity. Scale bar, $25 \mu \mathrm{m}$. B. Bundle of immunoreactive fibers (arrowhead) from ventral midline somata that run in VCII of the mesothoracic ganglion. Scale bar, $50 \mu \mathrm{m}$.

sufficient to eliminate all staining. Tracts within the ganglia were named according to the scheme used by Tyrer and Gregory (1982).

Physiology. Locusts were mounted ventral surface uppermost, with the distal segments of all legs free to move. The meso- and metathoracic ganglia were stabilized on a wax-coated stainless steel platform and the thorax perfused with a constant flow of locust saline at a rate of $3 \mathrm{ml} /$ min and at a temperature of $20^{\circ} \mathrm{C}$. Into this perfusate, known concentrations of drugs (picrotoxin or bicuculline; Sigma) could be introduced by means of a mixing device located close to the locust with a dead space of $100 \mu \mathrm{l}$. To reduce possible movements of the ganglia that might result from twitches of the thoracic muscles during the long-term impalement of a neuron, the connectives anterior to the mesothoracic ganglion and posterior to the metathoracic ganglion were cut, as were the lateral nerves, except nerves 5 . The sheath on the ventral surface of the metathoracic ganglion was treated with a $1 \%(\mathrm{wt} / \mathrm{vol})$ solution of protease (Sigma type XIV) in saline for $2 \mathrm{~min}$. Microelectrodes filled with $2 \mathrm{M}$ potassium acetate and with DC resistances of 50-80 M $\Omega$ were then driven across the softened sheath and into the somata of interneurons and motor neurons. Identification of the impaled motor neurons followed established procedures (Hoyle and Burrows, 1973). Local reflexes were evoked in two ways. First, the tibia was extended about the femur in controlled waveforms by a servomotor that was in turn driven by a function generator. This evokes an interjoint reflex involving the muscles that move the tarsus (Burrows and Horridge, 1974) and a resistance reflex involving the muscles that move the tibia. Second, spurs at the tibiotarsal joint were deflected upwards (Burrows and Pflüger, 1986) using a small glass probe. This evokes an inhibitory tactile reflex in the single excitatory motor neuron innervating the levator tarsi muscle. All recordings were stored on magnetic tape and displayed on a Gould ES 1000 recorder.

Dye-labeling of interneurons. Some spiking local interneurons that make direct inhibitory connections with the levator tarsi motor neuron were identified by simultaneous recording from both neurons. The interneuron was then filled iontophoretically with hexamminecobaltic chloride (Brogan and Pitman, 1981) using $500 \mathrm{msec}$ depolarizing current pulses of 5-10 nA at a frequency of $1 \mathrm{~Hz}$ for $10 \mathrm{~min}$. After a further 20 $\mathrm{min}$, the ganglion was dissected out and the stain developed with ammonium sulfide and intensified with silver (Pitman et al., 1972; Bacon and Altman, 1977). Interneurons were drawn from whole-mounts and then embedded in wax and sectioned transversely at $10 \mu \mathrm{m}$.

Spiking local interneurons were also labeled by intracellular injection of Lucifer yellow (Stewart, 1978). The ganglia were then processed for immunohistochemistry. Every second section was stained with the an- tibody so that it was possible to determine whether somata labeled with Lucifer yellow were also immunoreactive.

\section{Results}

\section{Immunocytochemistry}

Two populations of spiking local interneurons that receive direct input from mechanoreceptors on one leg have been identified in the thoracic ganglia of the locust (Siegler and Burrows, 1984; Burrows and Watkins, 1986). The cell bodies of one group lie at the ventral midline of the ganglia, and those of the other in bilaterally symmetrical populations in the anterior lateral cortex. Neurons in both groups have 2 distinct fields of branches, one ventral, the other dorsal, linked by a single neurite.

Ventral midline group. The cell bodies of these neurons are part of a population of about 200 that lies at the ventral midline, extending from VCI anteriorly to the somata of 2 common inhibitory motor neurons posteriorly (Siegler and Burrows, 1984). Their primary neurites run in ventral commissure II (VCII) and they have a prominent neurite in the perpendicular tract (PT) linking a ventral field of dense, fine branches to a dorsal field of sparse, varicose branches (Fig. 1) (Siegler and Burrows, 1984). VCII lies approximately midway between the anterior and posterior edges of the population. Somata posterior to VCII stain with the antibody (Fig. $2 A$ ) and are separated by a transverse line from those anterior to it, which do not stain (Fig. 6B). Estimates from serial sections indicate that approximately 50 cell bodies, with diameters of 15-30 $\mu \mathrm{m}$, are stained. This pattern is repeated in each of the 3 thoracic ganglia.

A bundle of stained neurites arises from the stained somata (Fig. $2 B$ ) and crosses to the contralateral side of the ganglion in VCII (Fig. 3). Stained neurites emerge from this commissure to enter the lateral part of the PT (Figs. $3 F, 4 A$ ). These morphological features, revealed by antibody staining, are typical of spiking local interneurons in this midline group (Fig. 1). No other neurons with these features are known. 

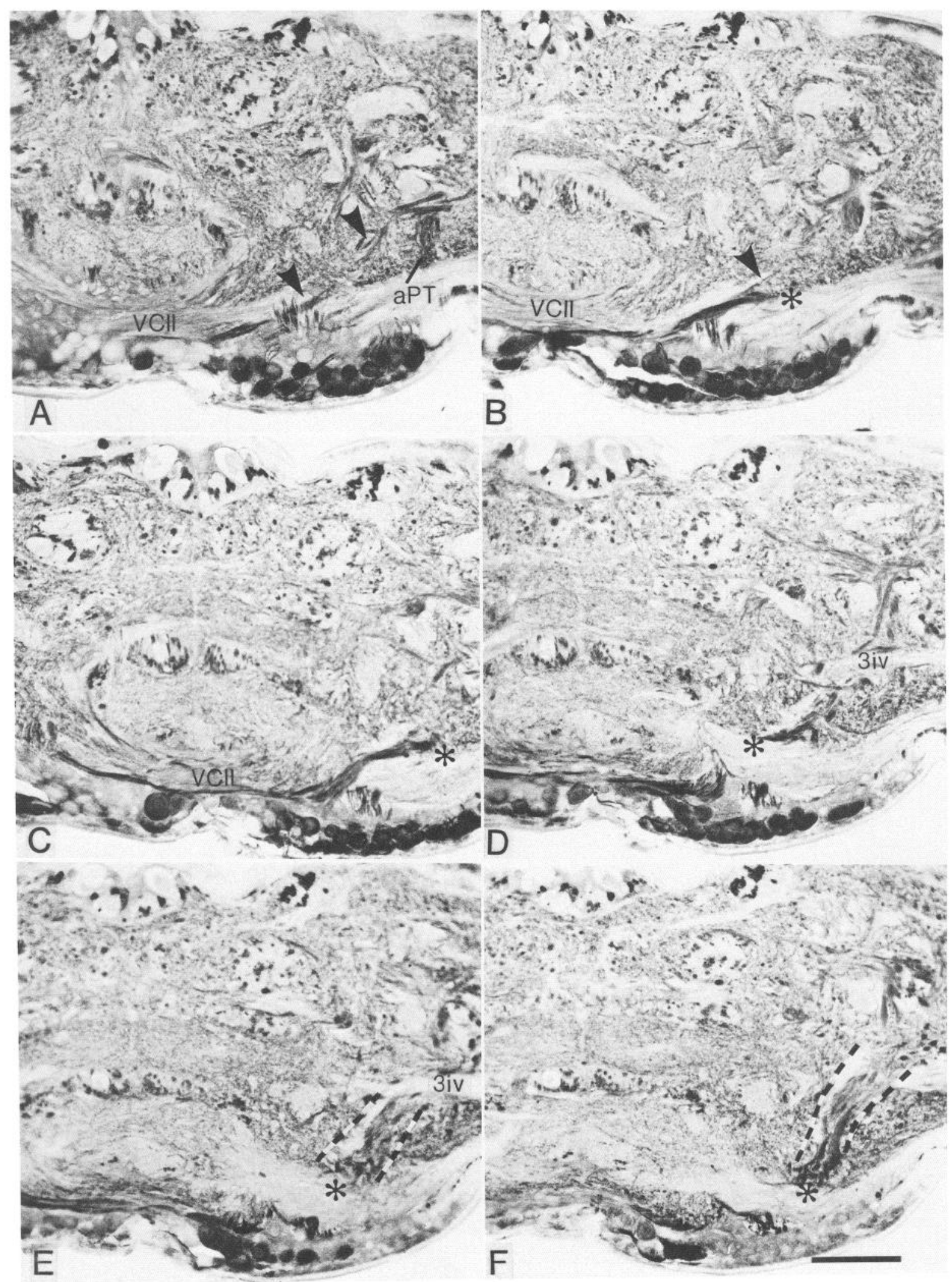

Figure 3. Six serial sections running anterior to posterior through VCII of the prothoracic ganglion, showing the path of neurites stained with the GABA antibody. $A, B$, Anterior bundle of stained neurites (arrowheads) emerges from $V C I I$ to run anteriorly in a dorsolateral path into the neuropile across the $a P T$. $C-F$, Larger bundle of stained neurites from VCII (asterisks) runs into the PT (broken lines, $E$ and $F$ ) anterior to the fourth root of nerve 3 (3iv). Scale bar, $100 \mu \mathrm{m}$. 


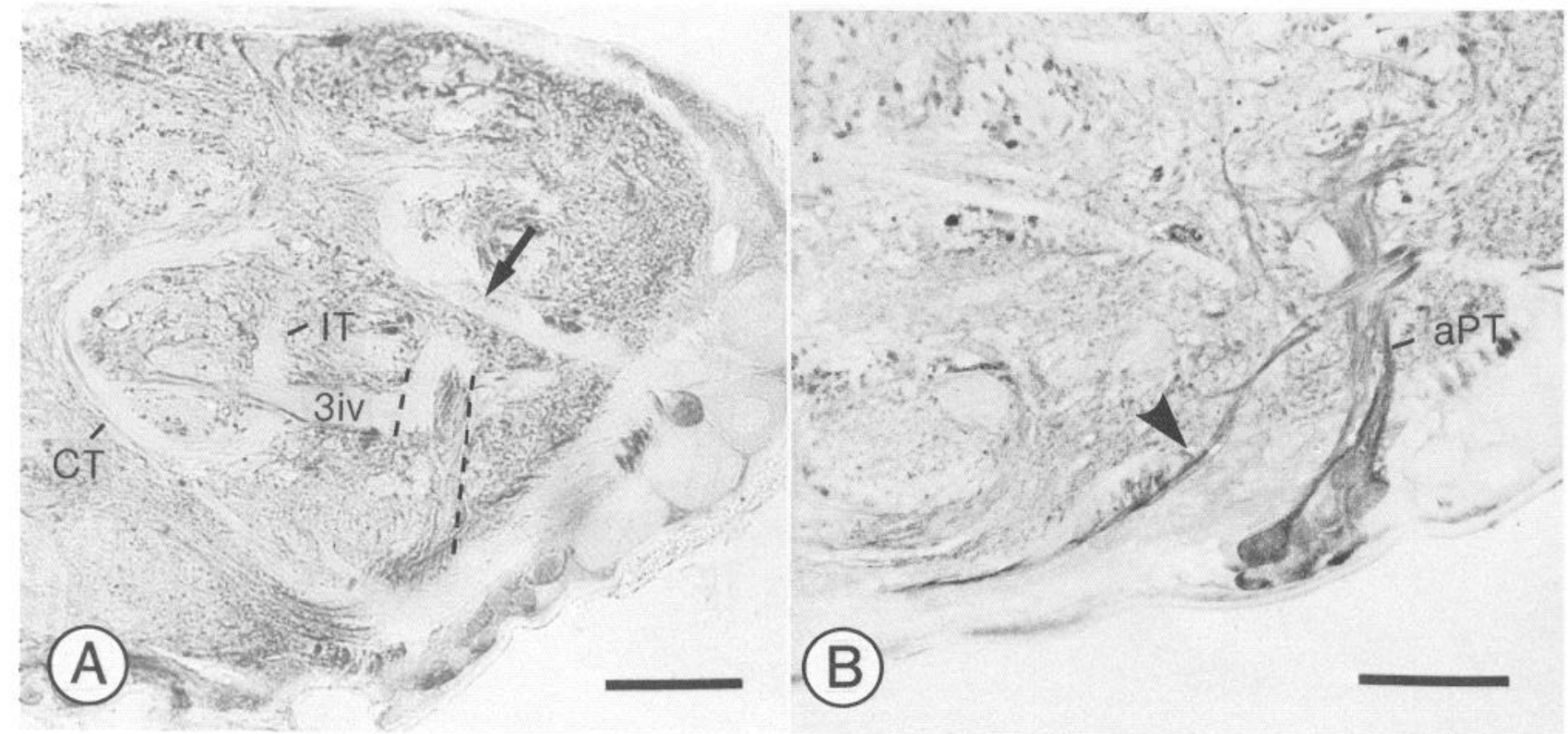

Figure 4. A, GABA antibody staining of processes in the PT (dashed lines) in the metathoracic ganglion. By contrast, processes in the "C" (CT) and "I" $(I T)$ tracts and the fourth root of nerve $3(3 \mathrm{iv})$ are all unstained. Arrow points to the primary neurite of the fast extensor tibiae motor neuron, which is also unstained. $B$, Anterior antibody-stained bundle of fibers (arrowhead) from VCII of the mesothoracic ganglion runs from the midline to cross anterior to the $a P T$. The aPT contains processes from a group of more lateral cell bodies that is also stained. Scale bars, $100 \mu \mathrm{m}$.

VCII, however, contains many processes that do not stain, and in the PT only neurites in the lateral part are stained (Fig. $4 A$ ). Furthermore, some of the stained neurites in VCII do not run to the PT and may thus belong to another group of neurons, with midline somata so far uncharacterized, or to sensory neurons. These stained neurites turn obliquely from VCII into the neuropile immediately anterior to the fourth root of nerve 3 (R3iv), $100-150 \mu \mathrm{m}$ from the midline (Figs. $3 A, 4 B$ ). The bundle passes just lateral to the ventral lateral tract before crossing in front of another stained tract (the anterior perpendicular tract; aPT) that runs dorsoventrally in the neuropile (Fig. $4 B$ ), carrying neurites from somata lateral to those of the midline group (see Watson, 1986).

Identity of stained somata. To demonstrate directly that spiking local interneurons of the midline group are stained with the antibody, the following procedure was adopted. The cell bodies of interneurons at the ventral midline of the meso- or metathoracic ganglion were impaled with microelectrodes filled with Lucifer yellow. Their receptive fields on a leg were determined by moving the joints and mechanically stimulating exteroreceptors such as hairs and campaniform sensilla. An interneuron positively identified in this way was then injected intracellularly with Lucifer yellow and the ganglion serially sectioned. Alternate sections stained with the antibody show that the somata of some spiking local interneurons exhibit GABA-like immunoreactivity (Fig. 5,A,B). These always lay posterior to VCII. The somata of other spiking local interneurons lying just ventral or anterior to VCII do not, however, stain (Fig. 5,C,D).

Anterior lateral group. The cell bodies of the anterior lateral group of spiking local interneurons are symmetrically arranged and lie just lateral to the entrance of the anterior connectives (Siegler and Burrows, 1984). Their primary neurites run in VCI and they have a prominent neurite in the oblique tract, which links a ventral field of dense, fine branches to a more dorsal field of sparse, varicose branches (Siegler and Burrows, 1984).
The nature of the postsynaptic effects mediated by these interneurons is as yet unknown. Somata in the cortex where these cell bodies are located do not show GABA-like immunoreactivity (Fig. $6 A$ ) and, moreover, processes in VCI do not stain (Fig. $6 B$ ). The lack of antibody staining of these interneurons is common to all 3 thoracic ganglia.

Reflex action of some spiking local interneurons can be blocked pharmacologically. If some of the spiking local interneurons in the midline group contain GABA, as is suggested by their GABAlike immunoreactivity, it might be expected that their inhibition of motor neurons could be abolished by blocking the effect of GABA. A consequence would be that certain reflexes would be abolished, as these interneurons form the central element of 3-neuron reflex arcs. Mechanosensory neurons make excitatory synapses on spiking local interneurons (Siegler and Burrows, 1983), which in turn make inhibitory synapses upon motor neurons (Burrows and Siegler, 1982). The following experiments test this hypothesis on 2 local reflexes by using drugs that antagonize the action of GABA.

An interjoint reflex. If the tibia is forcibly extended about the femur, then the tarsus undergoes a compensatory adjustment and is depressed about the tibia. The movement of the tarsus is caused by an excitation of some depressor tarsi motor neurons, but more reliably by an inhibition of the single levator tarsi motor neuron (Fig. 7). This motor neuron spikes spontaneously under normal physiological conditions, but the frequency of its spikes is modulated by prominent IPSPs. These are caused by 2 spiking local interneurons in the midline group that are themselves excited by tibial extension (Burrows and Siegler, 1982). Injecting depolarizing current into one of these interneurons induces spikes, each of which evokes an IPSP in the levator motor neuron with a consistent latency of less than $1 \mathrm{msec}$ (Fig. $7 A$ ). When the tibia is extended, the interneuron spikes and evokes a barrage of IPSPs that hyperpolarizes the motor neuron and abolishes its spikes (Fig. 7B). The inhibition of the motor 


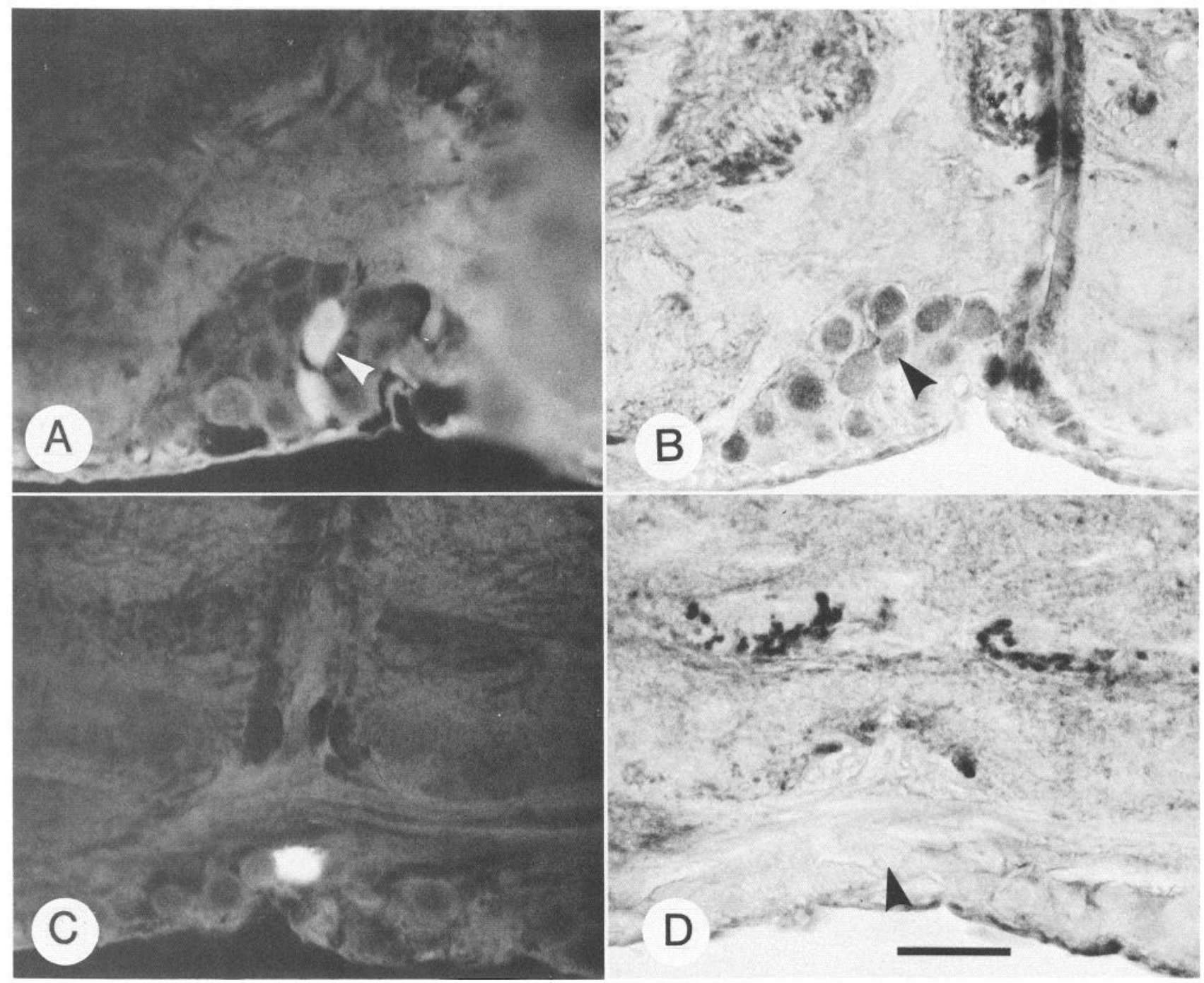

Figure 5. A,B, Double-labeling of the cell body of a mesothoracic spiking local interneuron. A, Cell body labeled with Lucifer yellow (arrowhead). $B$, In the adjacent section, the same cell body is stained with the antibody (arrowhead). Cell body of a metathoracic spiking local interneuron labeled with Lucifer yellow $(C)$ is not stained with the antibody on an adjacent section (arrowhead, D). Scale bar, $50 \mu \mathrm{m}$.

neuron is reliable and, provided that the spiking local interneurons spike, occurs whenever the tibia is extended. If the spikes of these interneurons are reduced in frequency or suppressedfor example, by injecting a steady hyperpolarizing current into one interneuron (the other interneuron will continue to spike), or by mechanical stimulation of particular exteroreceptors-the reflex effect in the motor neuron is greatly reduced (Fig. 7C). The interneuron in Figure 7 has an inhibitory region of its receptive field on the ventral tarsus. Thus, touching the ventral tarsus while extending the tibia through the same angle now causes only one spike in the impaled interneuron and results in only a weak inhibition of the motor neuron, presumably through a few spikes in the other local interneuron (Fig. 7C).

A similar reduction or abolition of the inhibitory reflex effect on the levator tarsi motor neuron can be achieved by the introduction of picrotoxin at a concentration of $10^{-4} \mathrm{M}$ into the saline bathing the thorax and the sheathed ganglia (Fig. 8). Picrotoxin is a noncompetitive blocker of GABA-mediated action. At the start of the experiment, each imposed extension of the tibia results in a high frequency of IPSPs that suppress the tonic sequence of spikes in the motor neuron (Fig. $8 A$ ). Typically the action of picrotoxin becomes apparent after about 5-10 min (Fig. $8 B$ ). In the example shown, IPSPs are still visible after 9 min both during the imposed movement and, less frequently, during the tonic spiking that consequently rises in frequency. After $18 \mathrm{~min}$, however, no IPSPs accompany the movement or interrupt the now high tonic frequency of spikes, and the reflex is completely abolished (Fig. 8C).

The removal of picrotoxin from the saline allows the reflex to recover (Fig. 8,D-F). In the example shown, the picrotoxin was removed after $17 \mathrm{~min}$ of application. Thirty-three minutes after the initial application, IPSPs began to reappear and to reduce the frequency of spikes during the imposed movement of the tibia (Fig. 8D). After $37 \mathrm{~min}$, the IPSPs become more numerous and of greater amplitude (Fig. $8 E$ ) until after $50 \mathrm{~min}$, recovery is complete (Fig. $8 F$ ). The reflex is now as effective as at the start. The same sequence of blockage and recovery of the reflex was observed in 6 locusts.

Abolition of the compensatory reflex was also achieved by using $5 \times 10^{-5} \mathrm{M}$ picrotoxin, but the effect took longer to appear. A $10^{-5} \mathrm{M}$ solution, however, failed to produce a change after $30 \mathrm{~min}$ and a $10^{-6} \mathrm{M}$ solution was without effect after $55 \mathrm{~min}$. 


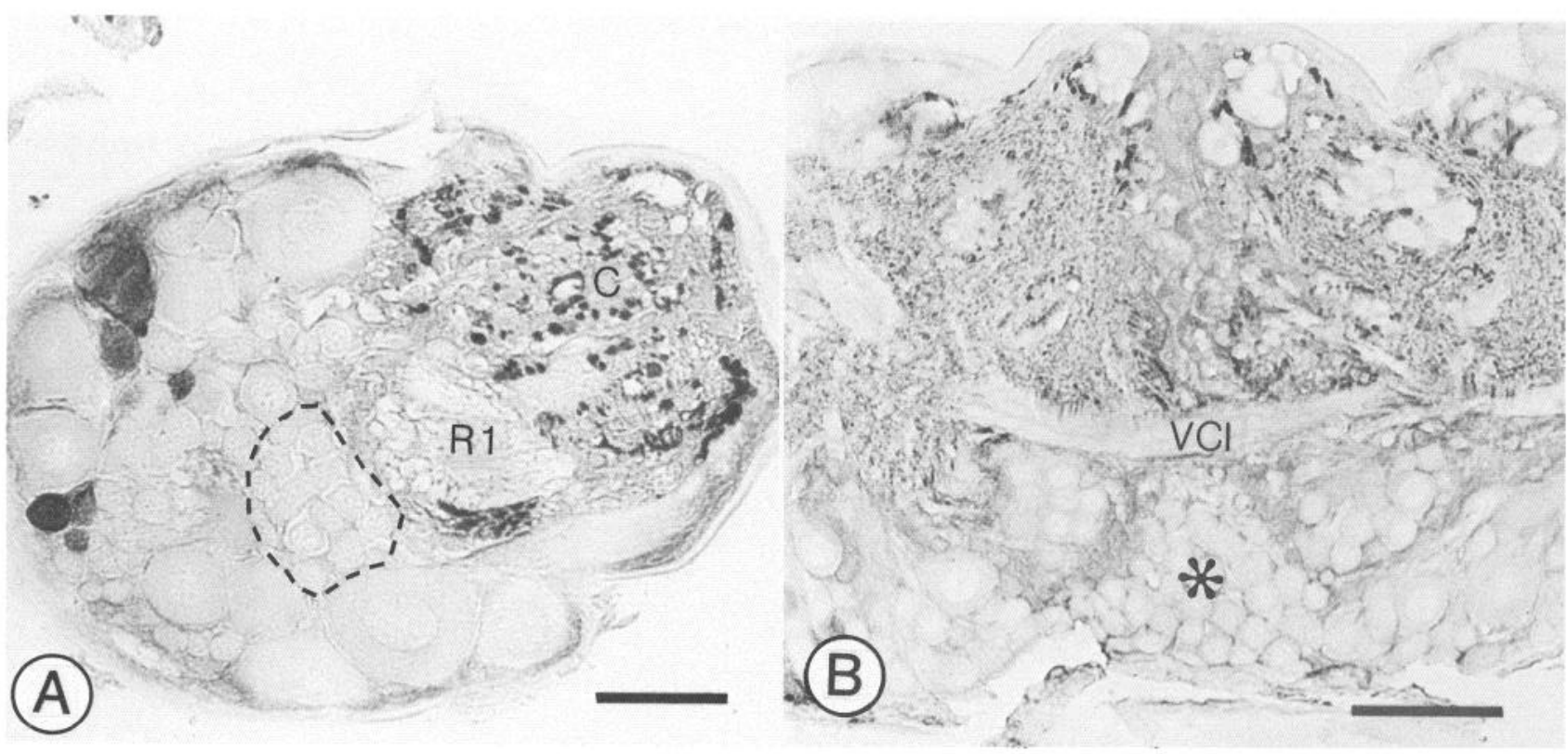

Figure 6. A, anterior lateral group of spiking local interneurons. The region of the ventral cortex of the mesothoracic ganglion occupied by cell bodies of these interneurons (outlined by dashed line) does not stain with the antibody. Some processes in the connective $(C)$ are stained, but none are stained in a root of nerve $1(R I)$. A few cell bodies belonging to a lateral anterior group of neurons (Watson, 1986) are stained on the left of the section. Scale bar, $50 \mu \mathrm{m} . B$, VCI in the prothoracic ganglion contains no stained neurites. Ventral to this commissure and anterior to $V C I$ is a cluster of unstained somata (asterisk), some of which belong to spiking local interneurons of the midline group. Scale bar, $100 \mu \mathrm{m}$.
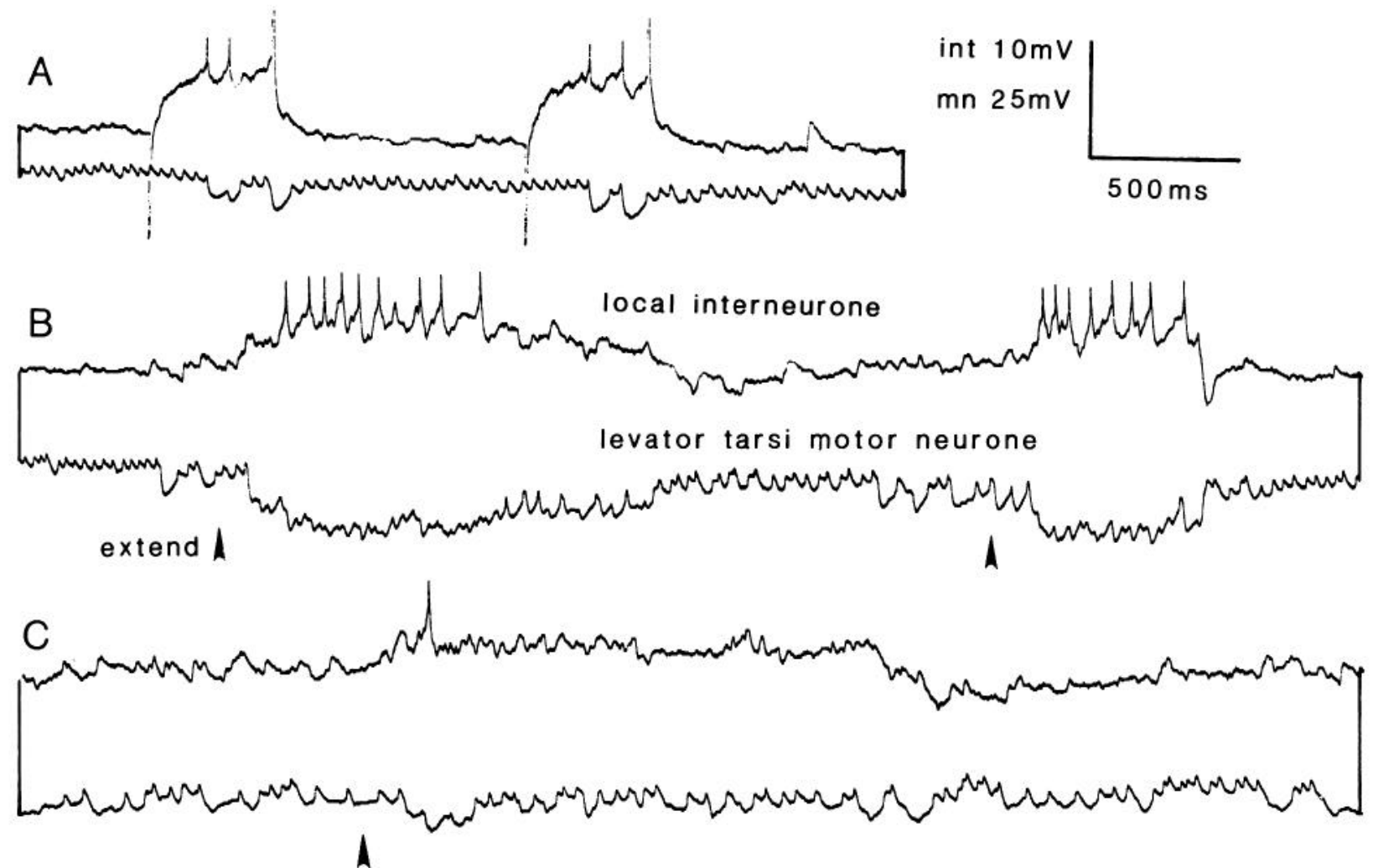

Figure 7. Spiking local interneuron in the metathoracic ganglion inhibits a motor neuron and controls a local reflex. $A$, Depolarizing the interneuron to make it spike evokes large IPSPs in the single levator tarsi motor neuron. $B$, Local interjoint reflex is elicited by forcibly extending the tibia: The interneuron spikes and inhibits the tonic discharge of the motor reuron. $C$, The movement is repeated but is now accompanied by stimulation of the inhibitory region of the interneuron's receptive field. All but one of its spikes are suppressed, with the result that the inhibition of the motor neuron is greatly reduced. The reflex therefore fails, demonstrating the importance of the spiking local interneurons in this pathway. 


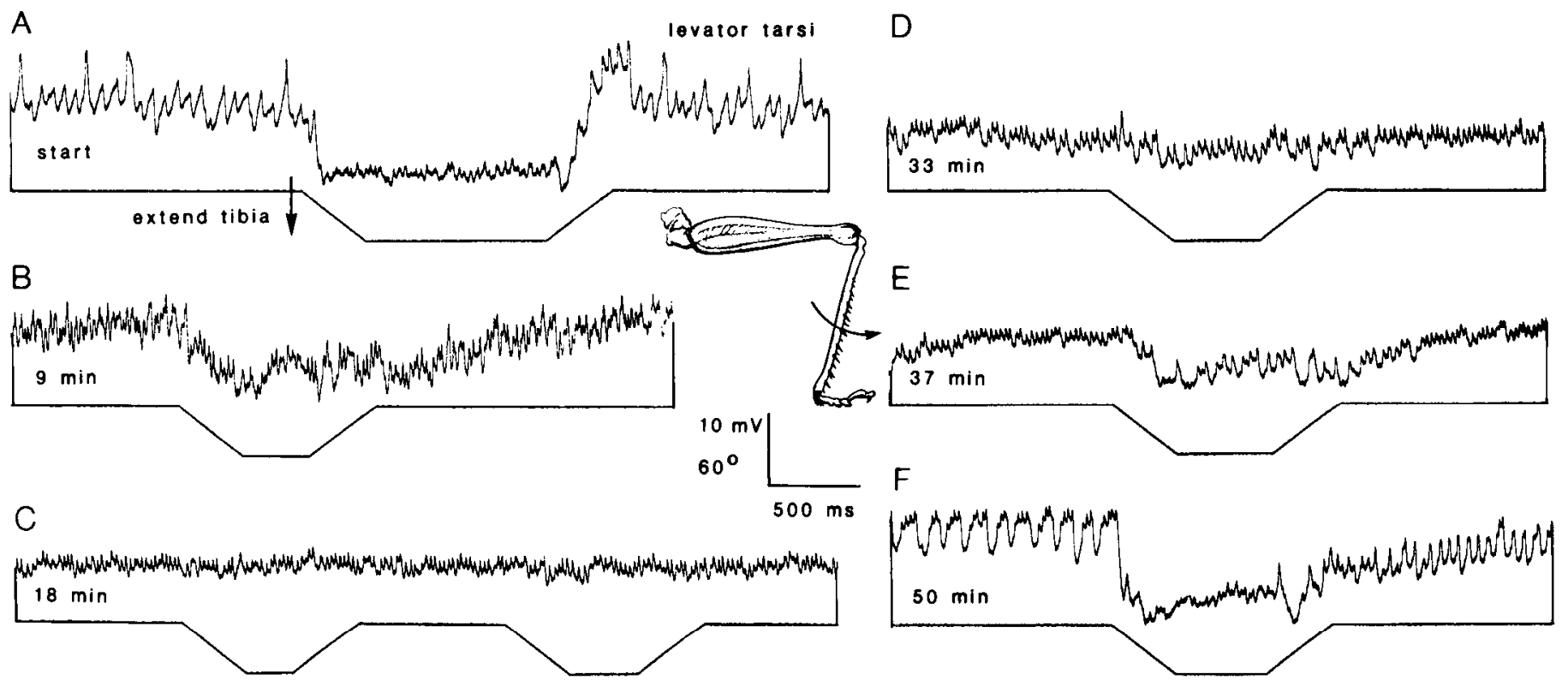

Figure 8. Effects of $10^{-4}$ picrotoxin on a local interjoint reflex of the levator tarsi motor neuron in a hind leg. The tibia was extended at a constant velocity through an angle of $40^{\circ}$, as indicated in the diagram and shown on the lower trace. $A$, Reflex before picrotoxin was applied. Intracellular recordings from the levator tarsi motor neurone show a high frequency of IPSPs during the imposed movement. $B$, Reduction of the reflex inhibition 9 min after application of picrotoxin. $C$, Abolition of the reflex inhibition after $18 \mathrm{~min}$. The picrotoxin was removed after 17 min. $D$, Partial recovery of the reflex after $33 \mathrm{~min}$. $E$, Further recovery after $37 \mathrm{~min}$. $F$, Complete recovery after $50 \mathrm{~min}$.

Bicuculline, which can act as a competitive blocker of the action of GABA, had no effect on the occurrence of the IPSPs even when applied for $60 \mathrm{~min}$ at concentrations of $5 \times 10^{-4} \mathrm{M}$.

The same imposed movement of the tibia about the femur that evokes the compensatory reflex movement of the tarsus also evokes a resistance reflex in muscles of the femur. An imposed extension of the tibia thus leads to an excitation of flexor tibiae motor neurons and an inhibition of extensor tibiae ones, so that the imposed movement is opposed. The application of $10^{-4} \mathrm{M}$ picrotoxin is without effect on the reflex excitation of the flexor motor neurons throughout the observed blockage of the inhibitory effect on the levator tarsi motor neuron. This shows that the sensory inputs continue to reach the ganglion and exert their normal effect at the first central synapses.

$A$ tactile reflex. An upward deflection of a spur at the tibiotarsal joint reflexly inhibits the levator tarsi motor neuron. The single afferent at the base of a spur synapses directly upon spiking local interneurons (Burrows and Pflüger, 1986). Afferents from the 2 anterior spurs excite interneurons other than those excited by the 2 posterior ones, but movement of all 4 spurs inhibits the levator tarsi motor neuron (Fig. 9, $A, B$ ). Hyperpolarizing one of these interneurons to abolish its spikes reduces the reflex inhibition of the levator motor neuron (Burrows and Pflüger, 1986). The reflex is not completely abolished by this procedure because more than one interneuron with inputs from the spurs converge onto the motor neuron.

The addition of picrotoxin, but not bicuculline, to the saline also blocks this inhibitory reflex. Ten minutes after the application of $10^{-4} \mathrm{M}$ picrotoxin, the inhibition resulting from touching the anterior (Fig. 9C) or posterior (Fig. 9D) spurs is reduced. After 14 min the inhibition is completely abolished (Fig. 9, $E, F$ ). After $17 \mathrm{~min}$ the picrotoxin was removed, with the result that some recovery of the reflex is apparent after $25 \mathrm{~min}$ (Fig. 9, $G, H$ ). Complete recovery occurs after $35 \mathrm{~min}$ so that the reflex effects are indistinguishable from those at the start even though the frequency of spikes in the motor neuron is now higher (Fig. $9, I, J)$.

\section{Discussion}

GABA is implicated as an inhibitory transmitter at insect neuromuscular junctions and acts by increasing chloride conductance (Usherwood and Grundfest, 1965; Usherwood and CullCandy, 1975). GABA also hyperpolarizes unidentified neurons in the sixth abdominal ganglion of the cockroach (Kerkut et al., 1969a, b; Walker et al., 1971) and motor neurons in the metathoracic ganglion (Pitman, 1971) when applied iontophoretically onto their somata. In the sixth abdominal ganglion the hyperpolarization has the same reversal potential as do IPSPs induced by electrical stimulation of the genital nerve, and both are reversed by bathing in chloride-free saline (Pitman and Kerkut, 1970; Pitman, 1971).

Here, immunocytochemical and pharmacological experiments show that some spiking local interneurons are labeled by an anti-GABA antibody and that the inhibition of specific motor neurons caused by certain interneurons during reflex movements is blocked by picrotoxin. The antibody stains somata in part of the cluster of midline somata that contains spiking local interneurons involved in the reflexes studied. Also stained are ventral commissure II, which carries the primary neurites, and the lateral part of the perpendicular tract, which carries the prominent process linking the dorsal and ventral fields of branches. Only spiking local interneurons with receptive fields on one leg are known to have this characteristic morphology. Furthermore, labeling individual interneurons with I ucifer yellow after careful physiological characterization showed clearly that some spiking local interneurons in this group exhibit GABAlike immunoreactivity.

To demonstrate directly that the interneurons that synapse onto the levator tarsi motor neuron are labeled by the antibody requires the following experiment. Simultaneous recordings 


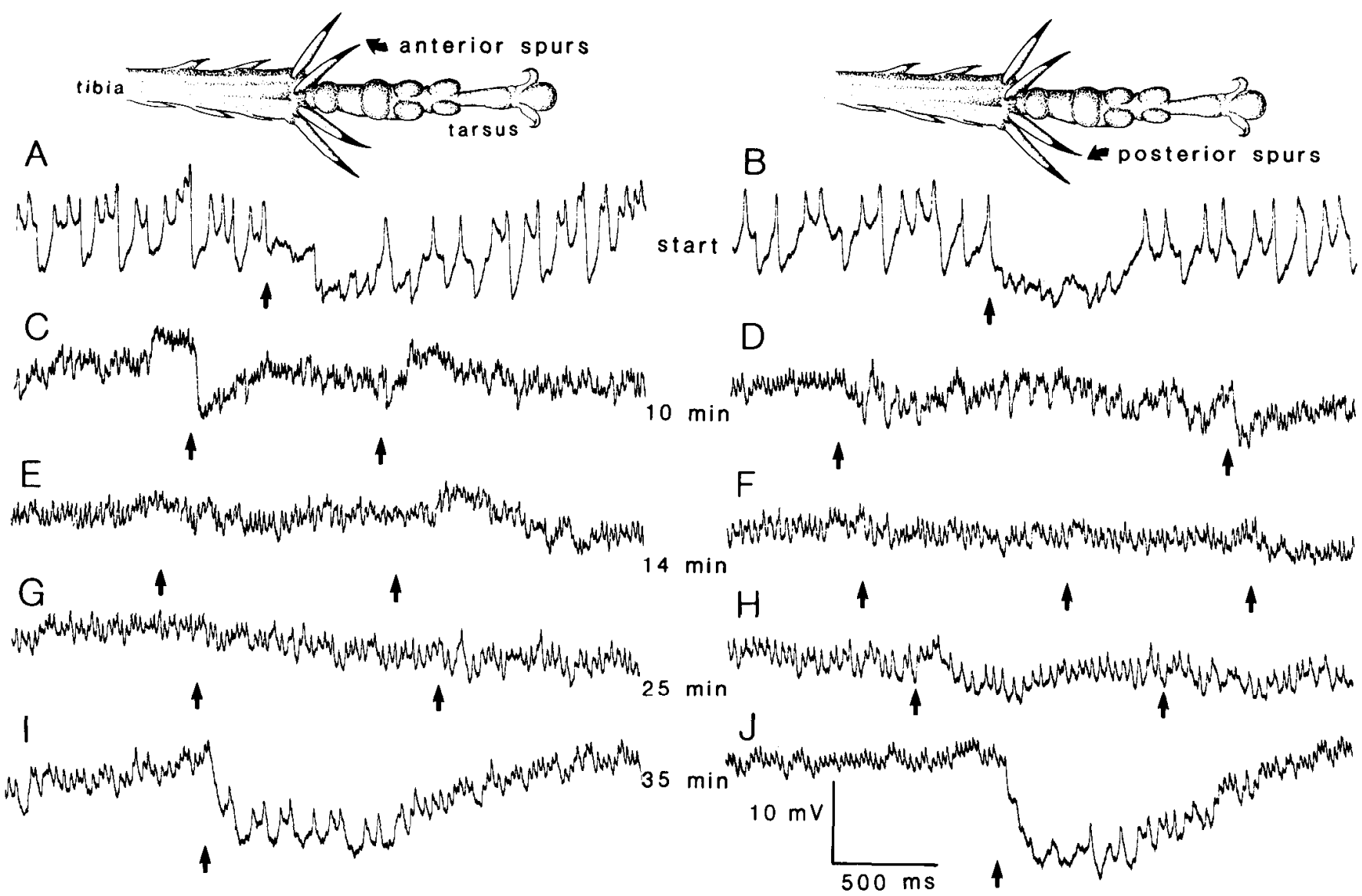

Figure 9. Effects of $10^{-4}$ picrotoxin on a tactile reflex. Anterior (left-hand column) or posterior (right-hand column) spurs were deflected upwards with a glass probe while an intracellular recording was made from the levator tarsi motor neuron. $A, B$, The reflex inhibition before application of picrotoxin. $C, D$, Reduction of the reflex after $10 \mathrm{~min} . E, F$, Abolition of the reflex after $14 \mathrm{~min}$. The picrotoxin was removed after 17 min. $G, H$, Partial recovery of the reflex after $25 \mathrm{~min}$. $I, J$, Complete recovery of the reflex after $35 \mathrm{~min}$.

would need to be made from the 2 neurons to demonstrate an inhibitory connection (see Fig. 7), and the interneuron stained with Lucifer yellow. Then alternate sections would be processed for immunocytochemistry (see Fig. 5) to show that the same cell body is labeled by both methods. This would be an exceptionally difficult experiment, as double recordings from these neurons have been obtained on only 8 occasions during more than 5 years' work on these spiking local interneurons.

To be certain about the interpretation of the antibody staining and the pharmacological disruption of the inhibitory reflex effects of the interneurons, it is necessary to know, first, the specificity of the antibody and, second, the detailed pharmacological properties of picrotoxin and bicuculline.

\section{Antibody specificity}

The specificity of the antibody has been well characterized in vitro and shows a low cross-reactivity ratio with other amino acids and related compounds that are possible transmitters in insects (Seguela et al., 1984). Free GABA-peptide conjugates will readily displace radiolabeled GABA-peptide already bound to the antibody, but similar conjugates of glycine are only 1:795 as effective and conjugates of taurine $(1: 17,700)$, aspartate, and glutamate $(<1: 50,000)$ are even less effective (Seguela et al., 1984). In control experiments, all antibody staining was eliminated by preincubation of the antiserum with a GABA-BSA conjugate. The best demonstration of the specificity of the an- tibody, however, is its ability to stain the somata, primary neurites, and axons of the 3 common inhibitory motor neurons that innervate leg muscles (Watson, 1986). The common inhibitory motor neurons contain the enzyme glutamic acid decarboxylase (Emson et al., 1974), which catalyzes the synthesis of GABA from glutamate, and their neuromuscular effects are mimicked by direct application of GABA (see Usherwood and Cull-Candy, 1975). The axons of the common inhibitor neurons are the only ones stained in nerves 3,4 , and 5 . The antibody, therefore, fails to stain the excitatory leg motor neurons, which are probably glutamatcrgic (Usherwood and Cull-Candy, 1975), the dorsal unpaired median neurons, which contain octopamine (Evans and O'Shea, 1977, 1978), and sensory neurons.

\section{Action of picrotoxin and bicuculline}

In our experiments picrotoxin, but not bicuculline, reversibly blocked the inhibition in certain motor neurons caused by spiking local interneurons. Though picrotoxin is frequently used to block the action of GABA, it is a noncompetitive antagonist, probably acting on the chloride ionophore (Yarowsky and Carpenter, 1978; Iversen, 1984). In Aplysia neurons, both acetylcholine and GABA can activate chloride channels (Yarowsky and Carpenter, 1978) and at the crustacean neuromuscular junction glutamate can increase chloride conductance (Lingle and Marder, 1981). Glutamate can also act in this way in cultured cockroach neurons (Usherwood et al., 1980). 
In the vertebrate nervous system, bicuculline binds competitively with certain GABA receptors (Enna, 1983). In cockroaches, bicuculline methochloride is reported to inhibit the hyperpolarization of unidentified neurons brought about by iontophoresis of GABA onto their somata (Walker et al., 1971). While it might be argued that the lack of effect of bicuculline in the present study could be related to the relative instability or insolubility of the pure compound as compared with its quaternary salts, Lummis and Sattelle (1985) have found that bicuculline methiodide did not inhibit the binding of ${ }^{3} \mathrm{H}-\mathrm{GABA}$ to a putative GABA receptor in cockroach nerve cord extracts. The GABA receptor(s) described to date in insects show pharmacological differences from known GABA receptors in vertebrates. The failure of bicuculline to block the inhibition of motor neurons in the present study cannot, therefore, be construed as an argument against GABA being the transmitter released by the interneurons.

Taken together, the immunocytochemical and pharmacological experiments provide strong evidence that some spiking local interneurons contain GABA, and that the inhibition they evoke in levator tarsi motor neurons is mediated by GABA. It cannot be ruled out, however, that another transmitter that activates chloride channels is implicated. In this context, it should be noted that though some spiking local interneurons are not labeled by the GABA antibody, all of the output connections made by those with somata in the midline group have, so far, been found to be inhibitory (M. Burrows, unpublished observations).

\section{Transmitter expression in spiking local interneurons}

Spiking local interneurons in the midline group may have similar morphological and physiological characteristics but different cytochemical properties. Cytochemical heterogeneity in populations of morphologically similar neurons has also been reported in the vertebrate nervous system (see Nieuwenhuys, 1985). In the locust thoracic nervous system, the population of cell bodies within the midline group that is labeled by the GABA antibody is tightly knit and does not include any unlabeled cell bodies. Preliminary double-labeling experiments have shown that, in addition to the spiking local interneurons, this region of the ganglion also contains the cell bodies of nonspiking local interneurons and unidentified neurons whose neurites make up the anterior bundle arising from VCII (Burrows and Watson, unpublished observations). How does this arrangement arise during development? Are the cell bodies grouped according to transmitter phenotype rather than according to their physiological or morphological properties? Is the expression of a GABAcrgic phenotypc controlled by ccll lincagc or by cnvironmental factors? Although it has been possible to examine such questions in only a few systems, there is evidence for both genetic and environmental control.

In locust thoracic ganglia, the dorsal unpaired median neurons appear to arise from a single neuroblast (see Goodman, 1982). The group consists of nonspiking local interneurons (Goodman el al., 1980) and spiking neurons innervating muscles (Hoyle, 1978; Watson, 1984). Many, if not all, contain octopamine (Evans and O'Shea, 1977, 1978; Goodman et al., 1979; Goodman, 1982), arguing for the influence of cell lineage. Not all neuroblasts, however, produce progeny containing the same transmitter. In the locust thoracic and abdominal nervous system, the most prominent of the small number of serotonergic neurons arise from a single neuroblast that produces 6 progeny (Taghert and Goodman, 1984). In a segment-specific pattern, 1, 2, or 3 of these descendants show serotonin-like immunoreactivity, but the others do not. Those that do are always the eldest. It is argued that, because serotonin immunoreactivity appears before the axons of the neurons reach their final targets, and because intersegmental differences in serotonin expression arise before the neurons exhibit any morphological differences, cell interactions are unlikely to be a controlling factor (Taghert and Goodman, 1984).

In the vertebrate central nervous system, however, environmental factors clearly influence the production of particular neurotransmitters (see Jonakait and Black, 1986) and changes in the transmitter expressed can apparently take place during normal development (Furshpan et al., 1976; Landis and Keefe, 1983; Jonakait and Black, 1986).

There are several ways in which the pattern of neurons that are labeled with GABA antibody could arise in locust thoracic ganglia. Studies on the development of spiking local interneurons which might shed some light on these processes are currently in progress.

\section{References}

Bacon, J. P., and J. S. Altman (1977) A silver intensification method for cobalt-filled neurones in wholemount preparations. Brain Res. 138: 359-363.

Bishop, C. A., and M. O'Shea (1982) Neuropeptide proctolin immunocytochemical mapping of neurons in the central nervous system of the cockroach. J. Comp. Neurol. 207: 223-238.

Brogan, R. T., and R. M. Pitman (1981) Axonal regeneration in an identified insect motoneurone. J. Physiol. 319: 34P-35P.

Burrows, M. (1975) Monosynaptic connexions between wing stretch receptors and flight motoneurones of the locust. J. Exp. Biol. 62: 189219 .

Burrows, M. (1985) The processing of mechanosensory information by spiking local interneurons in the locust. J. Neurophysiol. 54: 463478.

Burrows, M. (1987) Parallel processing of proprioceptive signals by spiking local interneurons and motor neurons in the locust. J. Neurosci. 7: 1064-1080.

Burrows, M., and G. A. Horridge (1974) The organization of inputs to motoneurones of the locust metathoracic leg. Phil. Trans. R. Soc. Lond. [Biol.] 269: 49-94.

Burrows, M., and H. J. Pflüger (1986) Processing by local interneurons of mechanosensory signals involved in a leg reflex of the locust. $\mathbf{J}$. Neurosci. 6: 2764-2777.

Burrows, M., and M. V. S. Siegler (1982) Spiking local interneurons mediate local reflexes. Science 217: 650-652.

Burrows, M., and M. V. S. Sicgler (1984) The morphological diversity and receptive fields of spiking local interneurones in the locust metathoracic ganglion. J. Comp. Neurol. 224: 483-508.

Burrows, M., and B. L. Watkins (1986) Spiking local interneurones in the mesothoracic ganglion of the locust: Homologies with metathoracic interneurones. J. Comp. Neurol. 245: 29-40.

Emson, P. C., M. Burrows, and F. Fonnum (1974) Levels of glutamate decarboxylase, choline acetyltransferase and acetylcholinesterase in identified motor neurones of the locust. J. Neurobiol. 5: 33-42.

Enna, S. J. (ed.) (1983) The GABA Receptors, Humana Press, Clifton, NJ.

Evans, P. D., and M. O'Shea (1977) The identification of an octopaminergic neurone which modulates neuromuscular transmission in the locust. Nature 270: 257-259.

Evans, P. D., and M. O'Shca (1978) The identification of an octopaminergic neurone and the modulation of a myogenic rhythm in the locust. J. Exp. Biol. 73: 235-260.

Furshpan, E. J., P. R. MacLeish, P. H. O'Lague, and D. D. Potter (1976) Chemical transmission between rat sympathetic neurons and cardiac myocytes developing in microcultures: Evidence for cholinergic, adrenergic, and dual-function neurons. Proc. Natl. Acad. Sci. USA 73: $4225-4229$.

Goodman, C. S. (1982) Embryonic development of identified neurons in the grasshopper. In Neuronal Development, N. C. Spitzer, ed., pp. 171-211, Plenum, New York. 
Goodman, C. S., M. O'Shea, R. McCaman, and N. C. Spitzer (1979) Embryonic development of identified neurones: Temporal pattern of morphological and biochemical differentiation. Science 204:12191222.

Goodman, C. S., K. G. Pearson, and Spitzer N. C. (1980) Electrical excitability: A spectrum of properties in the progeny of a single embryonic neuroblast. Proc. Natl. Acad. Sci. USA 77: 1676-1680.

Hoyle, G. (1978) The dorsal unpaired median neurons of the locust metathoracic ganglion. J. Neurobiol. 9: 43-57.

Hoyle, G., and M. Burrows (1973) Neural mechanisms underlying behavior in the locust Schistocerca gregaria. I. Physiology of identified motorneurons in the metathoracic ganglion. J. Neurobiol. 4: 3-41.

Iversen, L. L. (1984) Amino acids and peptides: Fast and slow chemical signals in the nervous system? Proc. R. Soc. Lond. [Biol.] 221: 245-260.

Jonakait, G. M., and I. B. Black (1986) Neurotransmitter phenotypic plasticity in the mammalian embryo. In Current Topics in Developmental Biology, vol. 20, T. S. Okada and H. Kendoh, eds., pp. 165175, Academic, London.

Kerkut, G. A., R. M. Pitman, and R. J. Walker (1969a) Iontophoretic application of acetylcholine and GABA onto insect central neurones. Comp. Biochem. Physiol. 31: 611-633.

Kerkut, G. A., R. M. Pitman, and R. J. Walker (1969b) Sensitivity of neurones in the insect central nervous system to iontophoretically applied acetylcholine or GABA. Nature 222: 1075-1076.

Landis, S. C., and D. Keefe (1983) Evidence for neurotransmitter plasticity in vivo: Developmental changes in properties of cholinergic sympathetic neurons. Dev. Biol. 98: 349-372.

Lingle, C., and F. Marder (1981) A glutamate-activated chloride conductance on a crustacean muscle. Brain Res. 212: 481-488.

Lummis, S. C. R., and D. B. Sattelle (1985) Insect central nervous system gamma aminobutyric acid receptors. Neurosci. Lett. 60:1318

Nieuwenhuys, R. (1985) Chemoarchitecture of the Brain, Springer, Heidelberg.

Pearson, K. G., R. K. S. Wong, and C. R. Fourtner (1976) Connexions between hair-plate afferents and motoneurones in the cockroach leg. J. Exp. Biol. 64: 251-266.

Pitman, R. M. (1971) Transmitter substances in insects: A review. Comp. Gen. Pharmacol. 2: 347-371.

Pitman, R. M., and G. A. Kerkut (1970) Comparison of the actions of iontophoretically applied acetylcholine and gamma amminobutyric acid with the EPSP and IPSP in cockrnach central neurones. Comp. Gen. Pharamacol. 1: 221-230.

Pitman, R. M., C. D. Tweedle, and M. J. Cohen (1972) Branching of central neurons: Intracellular cobalt injection for light and electron microscopy. Science 176: 412-414.
Seguela, P., M. Geffard, R. M. Buijs, and M. Le Moal (1984) Antibodies against gamma-aminobutyric acid: Specificity studies and immunocytochemical results. Proc. Natl. Acad. Sci. USA 81:38883892.

Siegler, M. V. S. and M. Burrows (1983) Spiking local interneurons as primary integrators of mechanosensory information in the locust. J. Neurophysiol. 50: 1281-1295.

Siegler, M. V. S., and M. Burrows (1984) The morphology of two groups of spiking local interneurones in the metathoracic ganglion of the locust. J. Comp. Neurol. 224: 463-482.

Siegler, M. V. S., and M. Burrows (1986) Receptive fields of motor neurons underlying local tactile reflexes in the locust. J. Neurosci. 6: $507-513$.

Sternberger, L. A. (1974) Immunocytochemistry, 2nd Ed., PrenticeHall, Englewood Cliffs, NJ.

Stewart, W. W. (1978) Functional connections between cells as revealed by dye-coupling with a highly fluorescent naphthalimide tracer. Ccll 14: 741-459.

Taghert, P. H., and C. S. Goodman (1984) Cell determination and differentiation of identified serotonin-immunoreactive neurons in the grasshopper embryo. J. Neurosci. 4: 989-1000.

Tyrer, N. M., and G. E. Gregory (1982) A guide to the neuroanatomy of locust suboesophageal and thoracic ganglia. Phil. Trans. R. Soc. Lond. [Biol.] 297: 91-123.

Usherwood, P. N. R., and S. G. Cull-Candy (1975) Pharmacology of somatic nerve-muscle synapses. In Insect Muscle, P. N. R. Usherwood, ed., pp. 207-280, Academic, London.

Usherwood, P. N. R., and H. Grundfest (1965) Peripheral inhibition in skeletal muscle of insects. J. Neurophysiol. 28: 497-518.

Usherwood, P. N. R., D. Giles, and C. Suter (1980) Studies of the pharmacology of insect neurones in vitro. In Insect Neurobiology and Pesticide Action (Neurotoxicology 79), pp. 115-128, Society of Chemical Industry, London.

Walker, R. J., A. R. Crossman, G. N. Woodruff, and G. A. Kerkut (1971) The effect of bicuculline on the gamma-aminobutyric acid (GABA) receptors of neurones of Periplaneta americana and Helix aspersa. Brain Res. 33: 75-82.

Watson, A. H. D. (1984) The dorsal unpaired median neurons of the locust metathoracic ganglion: Neuronal structure and diversity, and synapse distribution. J. Neurocytol. 13: 303-327.

Watson, A. H. D. (1986) The distribution of GABA-like immunoreactivity in the thoracic nervous system of the locust Schistocerca gregaria. Cell Tissue Res. 246: 331-341.

Yarowsky, P. J., and D. O. Carpenter (1978) A comparison of similar ionic responses to gamma-aminobutyric acid and acetylcholine. J. Neurophysiol. 4I: 531-541. 